\title{
Review of: "Intermittent fasting in the prevention and treatment of cancer"
}

\author{
Federico Bozzetti
}

Potential competing interests: The author(s) declared that no potential competing interests exist.

The paper "Intermittent Fasting in the Prevention and Treatment of Cancer " by Clifton et al. (1), written by a well-known group of experts in this field, is a well-done, comprehensive, updated review which summarizes the present status of knowledge of the role of the calorie restriction (not only the intermittent fasting as the title would suggest) in cancer prevention and treatment.

Since this topic is very broad it is not surprising that different issues (calorie restriction in cancer prevention in animals and in humans, calorie restriction in tumour control in animals and in humans, clinical studies versus biologic studies etc.....) are not fully balanced and findings referring to humans approximately cover one fourth of the overall text. The discrepancy between the abundance of experimental findings (which often look very promising) and the extreme paucity of positive data in the human experience could lead the reader to draw premature conclusions in favour of the calorie restriction in cancer patients. The authors, however, quite wisely, warn in their final recommendations against the indiscriminate of such policy. However, it should be clear to the reader that reducing the calorie intake and maintaining the body weight within the range of normal values is a safe and healthy habit which is effective in preventing not only cancer but cardiovascular diseases too, while the benefits of this practice in patients to control the tumour growth are far to be demonstrated and the practice of calorie restriction might also harm the patients.

The clinical experience is quite limited: the authors (1) mention 8 clinical studies (only 3 RCT) on fasting prior to chemotherapy, showing in 4 just the feasibility and safety of the approach and in 3 a lower toxicity. A prospective non-randomized study (2) reported that fasting $<13 \mathrm{~h}$ per night was associated with a $36 \%$ increased risk of breast cancer recurrence compared with those fasting for $\geq 13 \mathrm{~h}$ per night and a phase 2 RCT (3) reported the fasting-mimicking diet cohort more likely had a pathologic response, but at by protocol analysis, not at the intention-to-treat analysis.

I would add a few points which were not fully addressed in the paper:

1. In my opinion the most relevant information concerning the relationship between calorie restriction and tumour growth in humans comes from two studies $(4,5)$ in overweight/obese patients with prostate cancer who underwent a period of calorie restriction before surgery. In the first RCT (4), 40 patients were randomized either to receive their usual diet, or a regimen ranging from 16.6-14.5 kcal/kg/d during seven weeks to obtain weight loss of about $4.7 \mathrm{~kg}$. Contrary to the expectations (and compared with controls) the weight-losing arm showed significantly greater Ki67 proliferation rates at surgery. The 
second study (5) randomised 44 overweight/obese patients to hypocaloric regimen (minus 500-800 $\mathrm{kcal} / \mathrm{d}$ from their usual diet) to achieve $3.7 \mathrm{~kg}$ loss after 5-8 weeks and found no significant changes in both malignant epithelium apoptosis (TUNEL) or proliferation levels (Ki67). In keeping with these biologic findings, a double blind RCT evaluating increases in nutrient intake through administration of Anamorelin - a ghrelin analogue able to improve appetite in cachectic NSLC patients - reported a sharp increase in IGF-1 without any difference in survival at 4-month follow up of NSCLC patients compared to controls $(6,7)$. These findings are quite consistent with the authors conclusion (1) stating that " human studies evaluating the effects of intermittent fasting on growth signaling from insulin and other pertinent hormonal and inflammatory markers of carcinogenesis appear to be clinically insignificant, at least with the current data, which often lack statistical power and long-term follow-up".

2. There is a huge amount of literature showing that weight loss is an independent negative prognostic and predictive factor in cancer patients and the consequent present approach, recommended by the international societies of clinical nutrition, is to nutritionally support patients during their trajectory across intensive multimodal oncologic treatments including surgery.

3. The authors devoted a very short section to the issue of "Intermittent fasting and ketone formation". Whereas it is acknowledged that ketone bodies inhibit glycolysis and would explain why both fasting and ketogenic diet can potentially improve antioxidative defense mechanisms in normal tissues, but not in cancer cells which cannot utilize them because of mitochondrial defects, it is important to emphasize that a ketogenic diet is not necessarily hypocaloric but can be "normo-caloric". The use of high-fat, high-protein, low-glucose diet is a reasonable compromise to administer nutrients which are very well metabolized by the weight-losing host while are poorly utilized by the glucose-dependent cancer cells. The limited, sparse clinical experience of such approach has been recently summarized (8). In conclusion, I fully agree with the key message of the authors "we would recommend against intermittent fasting for patients undergoing active cancer treatment unless doing so as part of a clinical trial and we would also advise patients who previously used intermittent fasting as a weight-loss strategy before a cancer diagnoses not to continue it during treatment unless doing so as part of a clinical trial". A recommendation often muffled by the clamor of mass media, always at the never-ending research of sensational news regardless of their scientific validity, and neglected by some nutritionists who easily apply to cancer patients the same nutritional regimens against obesity which they are especially familiar with.

\section{REFERENCES}

1. Clifton KK, Ma CX, Fontana L, Peterson LL. Intermittent fasting in the prevention and treatment of 
cancer. CA Cancer J Clin. 2021 Aug 12. doi: 10.3322/caac.21694. Epub ahead of print. PMID: 34383300.

2. Marinac $\mathrm{CR}$, Nelson $\mathrm{SH}$, Breen $\mathrm{Cl}$, et al. Prolonged nightly fasting and breast cancer prognosis. JAMA Oncol. 2016;2:1049-1055.

3. de Groot S, Vreeswijk MP, Welters MJ, et al. The effects of short-term fasting on tolerance to (neo)adjuvant chemotherapy in HER2-negative breast cancer patients: a randomized pilot study. BMC Cancer. 2015;15:652.

4. Demark-Wahnefried W, Rais-Bahrami S, Desmond RA, Gordetsky JB, Hunter GR, Yang ES, Azrad M, Frugé AD, Tsuruta Y, Norian LA, Segal R, Grizzle WE. Presurgical weight loss affects tumour traits and circulating biomarkers in men with prostate cancer. Br J Cancer. 2017 Oct 24;117(9):1303-1313.

5. Henning SM, Galet C, Gollapudi K, Byrd JB, Liang P, Li Z, Grogan T, Elashoff D, Magyar CE, Said J, Cohen P, Aronson WJ. Phase II prospective randomized trial of weight loss prior to radical prostatectomy. Prostate Cancer Prostatic Dis. 2018 Jun;21(2):212-220.

6. Katakami N, Uchino J, Yokoyama T et al. Anamorelin (ONO-7643) for the treatment of patients with nonsmall cell lung cancer and cachexia: Results from a randomized, double-blind, placebo-controlled, multicenter study of Japanese patients (ONO-7643-04). Cancer 2018; 124: 606-616.

7. Takayama K, Katakami N, Yokoyama T et al. Anamorelin (ONO-7643) in Japanese patients with nonsmall cell lung cancer and cachexia: results of a randomized phase 2 trial. Support Care Cancer 2016; 24: 3495-3505.r breast cancer in the multicentre randomized phase 2 DIRECT trial. Nat Commun. 2020;11:3083.

8. Bozzetti F, Stanga Z. Does nutrition for cancer patients feed the tumour? A clinical perspective. Crit Rev Oncol Hematol. 2020 Sep;153:103061. doi: 10.1016/j.critrevonc.2020.103061. Epub 2020 Jul 12. PMID: 32777729. 